\title{
Promoting interdomain analogical transfer: When creating a problem helps to solve a problem
}

\author{
Ricardo A. Minervino ${ }^{1}$ - Valeria Olguín ${ }^{1}$ - Máximo Trench $^{2}$
}

Published online: 7 October 2016

(C) Psychonomic Society, Inc. 2016

\begin{abstract}
Research on analogical thinking has devised several ways of promoting an abstract encoding of base analogs, thus rendering them more retrievable during later encounters with similar situations lacking surface similarities. Recent studies have begun to explore ways of facilitating transfer at retrieval time, which could facilitate the retrieval of distant analogs learned within contexts that were not specially directed to emphasize their abstract structure. Such studies demonstrate that comparing a target problem to an analogous problem helps students retrieve base analogs that lack surface similarities. To devise more portable ways of enhancing analogical transfer, Experiment 1 replicated Kurtz and Loewenstein's (Memory \& Cognition, 35, 334-341, 2007) target-comparison procedure with an additional condition in which participants compared the target to a nonanalogous problem before attempting to reach its solution. Although comparing two analogous targets outperformed the standard transfer condition in promoting analogical transfer, comparing nonanalogous problems did not yield a transfer advantage. Based on prior studies that showed that the activity of creating analogous problems during their initial encoding elicits a more abstract representation of base analogs, in Experiment 2 we assessed whether constructing a
\end{abstract}

Electronic supplementary material The online version of this article (doi:10.3758/s13421-016-0655-2) contains supplementary material, which is available to authorized users.

Ricardo A. Minervino

minervinora@gmail.com

1 Psychology Department, University of Comahue, IPEHCS-CONICET-UNCo, Av. Argentina 1400, Box 95, 8300 Neuquen, Neuquen, Argentina

2 Psychology Department, University of Comahue, ECyC-IPEHCS-CONICET-UNCo, Bariloche, Rio Negro, Argentina second analogous target problem at retrieval time helps participants retrieve superficially dissimilar base analogs. As predicted, target invention increased the retrieval of distant sources. In both experiments we found an association between the quality of the generated schemas and the probability of retrieving a distant base analog from memory.

Keywords Analogy $\cdot$ Problem solving $\cdot$ Problem construction $\cdot$ Surface similarity $\cdot$ Retrieval

Thinking by analogy involves identifying a common relational system between two superficially dissimilar situations and generating further inferences driven by these commonalities (Gentner, 1983; Holyoak, 1984). In the most typical case of analogy, a familiar situation (the base analog) serves as a model by which one can comprehend a less familiar situation (the target analog).

Taking into account that analogy plays a central role in activities as diverse as learning and applying categories (Hofstadter \& Sander, 2013; Kurtz, Boukrina, \& Gentner, 2013), argumentation (Blanchette \& Dunbar, 2001), scientific discovery (Dunbar, 1995; Nersessian, 1984), decision making (Markman \& Moreau, 2001), teaching and learning (Gentner \& Gentner, 1983), and problem solving (Gick \& Holyoak, 1980, 1983), some researchers suggest that analogy is the core of human cognition (e.g., Gentner, 2003; Hofstader \& FARG, 1995).

Given the ubiquity of analogical thinking, it is not surprising that scholars have engaged in identifying the central difficulties that people face when making analogies, as well as in trying to design interventions to overcome them (see Gentner \& Smith, 2012; Holyoak, 2005, for revisions). One of the most robust findings in the experimental literature on analogical transfer is that people often fail to spontaneously retrieve analogous situations when they do not share surface features 
with the target situation being processed (Catrambone, 2002; Gentner, Rattermann, \& Forbus, 1993; Gick \& Holyoak, 1980; Holyoak \& Koh, 1987; Keane, 1987; Ross, 1984, 1987; for a discussion of apparently contradictory naturalistic findings, see Trench \& Minervino, 2015).

A considerable body of research has sought to enhance spontaneous analogical retrieval by means of promoting a more abstract encoding of the base analogs, so as to render them more accessible during later encounters with similar situations lacking surface similarities. Two successful strategies consisted in presenting the base analog together with its abstract schema (Goldstone \& Wilensky, 2008; Ross \& Kilbane, 1997) or with a second analogous situation (Catrambone \& Holyoak, 1989; Gick \& Holyoak, 1983; Ross \& Kennedy, 1990), and asking participants to compare them. More stripped-down interventions included making participants discuss the base analog with another student (Schwartz, 1995), explaining the problem to themselves (Ahn, Brewer, \& Mooney, 1992), or constructing a structurally equivalent problem (Bernardo, 2001a). When trying to promote transfer without asking participants to elaborate on the base situations, transfer advantages can still be obtained by means of eliminating irrelevant information (Goldstone \& Son, 2005) or by replacing domain-specific terms of the base situation with domain-general ones (e.g., replacing "typing" with "writing"; Clement, Mawby, \& Giles, 1994). What all these interventions have in common is the highlighting of the abstract structure of the base analogs. The shared assumption is that as future similar examples will have a stronger match with such more abstract representations than they would with specific examples having mismatching features, the retrieval of these relationally encoded base analogs will be more probable.

Despite the relative success of these interventions, a sensible question concerns whether improving people's initial encoding of examples is the only way to favor the retrieval of analogs. If changing the initial encoding is the only means for promoting analogical reminding, then we can give little help to someone currently searching for analogs in long-term memory. What we need in these cases are interventions to favor analogical reminding at the time of retrieval. To enhance the retrieval of base problems learned under conditions that were not purposely engineered to foster the extraction of their underlying abstract structure, Kurtz and Loewenstein (2007) and Gentner, Loewenstein, Thompson, and Forbus (2009) reasoned that as retrieval depends on the degree of match between the stored items and the memory probe, the beneficial effect of schema abstraction should also apply when elaborating on the target analog at retrieval time. To test this prediction, Kurtz and Loewenstein (2007) assessed the effectiveness of an intervention that consisted in providing participants with a second (unsolved) problem that was isomorphic to the target problem to be solved, and asking them to compare both problems prior to attempting their solution. As was the case with the "base comparison" interventions, this "target comparison" procedure resulted in enhanced transfer of the base solution as compared to the standard base-target condition. In subsequent work, Gentner et al. (2009) generalized the benefits of the target comparison strategy to autobiographic memories that were acquired several years before the experimental session, and also simulated the process of backward transfer using a retrieval algorithm and a set of stories that were developed before the late analogical encoding hypothesis was entertained. To carry out these simulations, Gentner et al. (2009) fed MAC/FAC (Forbus, Gentner, \& Law, 1995) with targets that consisted either in the original stories from the Karla the Hawk series of studies (Gentner et al., 1993) or in their respective abstract schemas, and had it run on a long-term memory comprising analogical matches, mere appearance matches, and several filler stories. MAC/FAC retrieved more analogical matches when using the schemas as working memory cues.

As suggested by the results of the target comparison studies, the process of late analogical abstraction opens a promising avenue for retrieving base situations learned within contexts that were not specially designed to highlight their underlying structures, and which may represent the vast majority of the situations we learn within and outside instructional settings (Loewenstein, 2010). In contrast to the widespread potential applicability of the late abstraction principle, the targetcomparison intervention still falls short of representing a truly portable cognitive strategy because participants depend on the provision of a second isomorphic target for every target problem they are to solve. The experiments reported in this study sought to replicate Kurtz and Loewenstein's (2007) original finding and to modify the original target-comparison task so as to help learners capitalize on the benefits of late abstraction, but without the need to be provided with a second analog target problem for each new target problem to be solved.

\section{Experiment 1}

The first purpose of Experiment 1 was to replicate Kurtz and Loewenstein's (2007) basic findings. With this objective, we included an experimental condition (comparing analogous targets; see Table 1 for a schematic presentation of the conditions of Experiment 1) comprising two phases: (1) a learning phase, in which participants received a base problem and its solution, and (2) a transfer phase, in which they had to solve a target analog after comparing it to a second unsolved target problem. Performance in this condition was compared with that of the standard transfer condition, in which participants had to solve the target problem after having read the base problem during the learning phase. To determine whether an eventual advantage of the experimental condition stemmed from the transfer of the learned procedure, and not merely 
Table 1 Problems and activities per condition in Experiment 1

\begin{tabular}{|c|c|c|c|}
\hline Condition & $\begin{array}{l}\text { Problems received during } \\
\text { learning phase }\end{array}$ & $\begin{array}{l}\text { Problems received during } \\
\text { transfer phase }\end{array}$ & Activity during transfer phase \\
\hline Standard transfer & The General & Radiation & Solving the Radiation problem \\
\hline Comparing analogous targets & The General & Radiation + The Fire Chief & $\begin{array}{l}\text { Comparing the target problems } \\
\text { and solving the Radiation problem }\end{array}$ \\
\hline $\begin{array}{l}\text { First principles control for } \\
\text { analogous targets }\end{array}$ & $\longrightarrow$ & Radiation + The Fire Chief & $\begin{array}{l}\text { Comparing the target problems } \\
\text { and solving the Radiation problem }\end{array}$ \\
\hline $\begin{array}{l}\text { Comparing disanalogous } \\
\text { targets }\end{array}$ & The General & Radiation + Weigh the asteroid & $\begin{array}{l}\text { Comparing the target problems and } \\
\text { solving the Radiation problem }\end{array}$ \\
\hline $\begin{array}{l}\text { First principles control for } \\
\text { disanalogous targets }\end{array}$ & 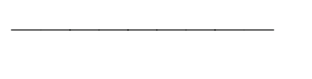 & Radiation + Weigh the asteroid & $\begin{array}{l}\text { Comparing the target problems and } \\
\text { solving the Radiation problem }\end{array}$ \\
\hline
\end{tabular}

from an enhanced comprehension of the target problem obtained as an outcome of the comparative process, the experimental condition was compared to a control condition (first principles control for analogous targets), in which the comparison between the two analogous targets was not preceded by the presentation of a base analog during the learning phase.

As suggested by the results of Kurtz and Loewenstein (2007), comparing the target problem to a second problem with a similar structure helps participants arrive at a more abstract representation of such problem, which in turn increases the retrieval of the critical analogous source. However, the question remains open as to the extent to which such an abstraction process necessarily requires the provision of a second target problem structurally similar to the first. In order to devise ways of promoting an abstract encoding of the target analog without providing a second analog, we speculated that having participants compare the target problem with a further nonisomorphic target might lead to the extraction of some abstract cues that could favor the retrieval of distant analogs. Our hypothesis was that the canonical partition of each of the problems in terms of its components (i.e., its goals, restrictions, operators, etc.) could frame the establishment of a set of correspondences between the (dissimilar) concepts that fill such slots in each of the problems. This could, in turn, result in conceptualizing commonalities and differences between those fillers, which could eventually result in appropriate cues for retrieving distant analogs. As an example, consider that someone has to face Duncker's (1945) Radiation problem, in which a patient has an inoperable tumor in his stomach. The tumor could be destroyed by certain kind of ray, but a beam of the required intensity would also destroy the surrounding tissues. Participants' task consists in devising ways of using the rays to destroy the tumor, but without harming the healthy tissues. Suppose that before attempting a solution, participants were asked to compare such problem with Duncker's (1945) Candle problem, in which they are supplied with candles and a matchbox-size box filled with tacks, with the challenge of fixing the candle to a wall so that once lit the wax would not drip onto the floor. Faced with the task of comparing both problems, an analysis of their respective restrictions could lead to considering them as two cases of "avoidance to damage near objects" (a structural similarity). On the other hand, an analysis of the permitted operators could lead to conceptualize them as "using a force versus using some office items to reach the objective" (an alignable difference). Given that similarities and differences of this kind tend to be more abstract than the particular elements in which they originate, we speculated that including them in a representation of the target problem could aid the retrieval of interdomain sources by means of providing additional and more appropriate cues for the memory search. In case it did, this alternative route to late abstraction would represent a more portable strategy than comparing the target to an externally provided analog because students could randomly choose any of a potentially indefinite number of problems to compare with the one to be solved.

Based on the above considerations, the second and separate objective of Experiment 1 was to determine if comparing a target with a nonanalogous problem could facilitate the retrieval of a previously received distant analog. Performance of the comparing disanalogous targets condition was compared with that of the standard transfer group, which was reused for this second purpose. To determine whether an eventual advantage of the experimental condition over the transfer condition stemmed from the transfer of the learned procedure, and not merely from an enhanced comprehension of the target problem obtained as an outcome of the comparative process, this experimental condition was compared with a control condition (first principles control for disanalogous targets), in which the comparison between the nonisomorphic targets was not preceded by the presentation of a base analog during the learning phase.

\section{Method}

Participants and design A total of 350 undergraduate students at University of Comahue volunteered to take part in the 
study and signed an informed consent for participation. They were randomly assigned to each of the five groups of the experiment (70 participants per group). The first block of three conditions (standard transfer, comparing analogous targets and first principles control for analogous targets) were associated to the purpose of replicating Kurtz and Loewenstein's (2007) finding about the efficacy of comparing two target analogs in retrieving a distant base analog. Combined with the standard transfer condition mentioned above, the two remaining conditions (comparing disanalogous targets and first principles control for disanalogous targets) served for the separated objective of determining the efficacy of comparing two nonisomorphic targets in retrieving a superficially dissimilar base analog. ${ }^{1}$ The dependent variable was the use of the base strategy during the solution of the target problem.

Procedure and materials All participants were interviewed at the Cognitive Studies Center of the University of Comahue. The number of participants per time slot ranged from four to six, with each participant working individually. During the learning phase of the standard transfer condition, participants (48 females and 22 males; $M_{\text {age }}=22.30, S D=2.58$ ) were asked to read three stories including a problem and its solution (see Table 1 for a synopsis of the conditions and procedures of the experiment). Each story was presented in a separate sheet of paper and was followed by the comprehension question "What critical insight allowed the problem in the story to be solved?" and by a space to provide a written answer. Participants were allowed to proceed to a subsequent page only after having read the preceding story and answered its corresponding question. Although the first and the third stories served as distracters, the second story was analogous to the target problem to be presented in the transfer phase. Taken from Gick and Holyoak (1980), the story The General told about a dictator who ruled a small country from a fortress located at its center, which could only be captured by a massive attack. A General had an army that was large enough to capture the fortress, but that could not be brought

\footnotetext{
${ }^{1}$ Although Experiment 1 includes five different conditions, it is not a five-condition design; it includes two blocks of conditions associated to separate objectives, being the baseline condition (i.e., standard transfer) reused in the second block. According to this, our data analysis will only compare each experimental condition to its specific control and to the baseline condition (common to both blocks), since any other comparison beyond these would lack theoretical interest and, worse yet, would be inappropriate to evaluate the efficacy of the interventions being tested. For example, to replicate Kurtz and Loewenstein's (2007) finding related to the advantages of comparing two analogous targets, this condition should result in a superior performance to that of the standard transfer condition and to that of the first principles control for analogous targets condition, but neither to that of the comparing disanalogous targets condition nor to that of first principles for disanalogous targets condition. Based on these considerations, the rejection criterion used for each of the two planned contrasts that correspond to each of the abovementioned blocks will involve splitting alpha by two: $\alpha=.025$.
}

into it through any of its radial roads, because they were mined and could explode if large groups of people passed over them. At the end of the story, the General figured out how to proceed: he divided his army into smaller groups and located each of them on a different road. The groups arrived at the fortress at the same time without detonating any of the mines (the original problems and the Spanish versions employed in the present study are presented in the supplemental materials). Participants were allotted $15 \mathrm{~min}$ to read the three stories and to answer their corresponding questions.

During a transfer phase, participants were asked to read Duncker's (1945) Radiation problem, presented in a separate sheet of paper. This problem told that a certain patient had an inoperable tumor in his stomach, which had to be destroyed to save the patient's life. Even though the patient's surgeon had a kind of ray that could destroy the tumor if applied with the necessary intensity, at such strength it would also destroy the surrounding tissues. Upon reading the problem, participants of the standard transfer condition were allotted $10 \mathrm{~min}$ to write down ways in which the rays could be used to destroy the tumor, but without harming the surrounding tissues.

The learning phase of the comparing analogous targets condition (43 females and 27 males; $M_{\text {age }}=22.69, S D=$ 3.10) was identical to that of the standard transfer condition. However, during the transfer phase, participants received a sheet of paper that included the tumor problem followed by an adapted version of The Fire Chief problem (Gick \& Holyoak, 1983). This second problem told about a large fire at a botanical garden, which could be suffocated by a highpressure water jet. After stating that such a powerful jet would also damage the exotic species of the garden, the problem asked for ways of using the water to extinguish the fire without harming the plants. Upon reading the Radiation and The Fire Chief problems, participants were asked to write down their main similarities and differences. After the 5 min allotted to the comparison task had elapsed, participants were allotted $10 \mathrm{~min}$ to write down different solutions to the Radiation problem.

As in Kurtz and Loewenstein (2007, Experiments 2 and 3), a control condition was employed to assess whether an eventual increase in the proportion of convergent solutions proposed by participants of the target-comparison condition was in fact mediated by the retrieval of the source story, as opposed to arising simply as a consequence of an improved comprehension of the target problem. Participants in this first principles control for analogous targets condition (46 females and 24 males; $M_{\text {age }}=23.31, S D=2.42$ ) received the same materials and tasks given to participants of the comparing analogous targets condition during the transfer phase, but without having received the base analog and the distracter stories during a prior phase of the experimental session. Taken collectively, this first block of three conditions was related to the objective of replicating Kurtz and Loewenstein's finding that 
comparing two analogous targets is beneficial for analogical retrieval.

The second and separate objective of Experiment 1 was associated with three conditions and consisted in assessing whether the retrieval of a distant base analog could also be improved by means of comparing the target analog to a nonisomorphic problem. The procedure followed in the comparing disanalogous targets condition (45 females and 25 males; $M_{\text {age }}=22.64, S D=2.02$ ) was identical to that of the comparing analogous targets condition, with the difference that the Radiation problem was now coupled with an adapted version of the Weigh the asteroid problem used by Chen, Mo, and Honomichl (2004). In the present version, four scientists arrive by boat to a remote island where a mysterious explosion has been recorded. They find a large asteroid and plan to load it on their boat, but one of the scientists reads from the safety warnings that the boat can safely carry up to $800 \mathrm{~kg}$. They have a scale in the boat, but it can weigh objects of at most $200 \mathrm{~kg}$, which is insufficient to weigh the asteroid. The problem asks participants for possible ways of using the extant scale to determine the weight of the asteroid, but without breaking it into smaller fragments.

As was done with the comparing analogous targets condition, a control condition was implemented to assess the extent to which an eventual increase in the proportion of convergent solutions to the Radiation problem given by participants who had compared unrelated targets was in fact mediated by the retrieval of the source story. Participants in the first principles control for disanalogous targets condition (41 females and 29 males; $M_{\text {age }}=23.06, S D=3.04$ ) received the same materials and tasks given to participants during the transfer phase of the comparing disanalogous targets condition, but without having received the critical base story and its solution during a prior phase of the experiment.

Scoring Two raters blind to condition scored whether any of the responses given by each participant to the Radiation problem involved a convergence solution. As in previous research using this problem, a response was scored as a convergence solution if it captured the principle of a multiplicity of lowintensity rays acting in concert on the tumor. Solutions involving repetition over time rather than in parallel were not counted as convergence solutions. Responses in which the application of rays at the same time is only implied were accepted. Raters agreed in $96 \%$ of the cases, and solved the cases of disagreement by discussion.

\section{Results and discussion}

A first set of analyses involving the comparing analogous targets, the standard transfer and first principles control for analogous targets conditions was carried out to assess whether the results from these conditions replicate the transfer advantage of comparing two analogous targets. Results showed that the type of transfer condition yielded an effect on the proportion of convergence solutions to the Radiation problem, $\chi^{2}(2, N=210)=15, p=.0006$. Planned comparisons using Bonferroni alpha corrections revealed that participants in the comparing two analogous targets condition outperformed those of the standard transfer condition in producing convergence solutions to the Radiation problem $(34.29 \%$ vs. $8.57 \%), \chi^{2}(1, N=140)=13.75, p=.0002, w$ $=.31$. This advantage does not reduce to an enhanced ability to solve the tumor problem by first principles, because participants of the comparing two analogous targets condition also outperformed participants who received the target comparison tasks without having received the base analog during a learning phase $(34.29 \%$ vs. $17.14 \%), \chi^{2}(1, N=140)=5.38, p=$ $.0204, w=.20$. Thus, comparing two target problems seems to produce a better retrieval probe to access the base story and its convergence solution. To better assess the mediating effect of late analogical abstraction on analogical retrieval, participants' success in extracting the structural features of the target analog during the comparison task was related to their performance in generating convergence solutions to the tumor problem. Participants were sorted as successful schema generators $(N=54)$ whenever they explicitly mentioned (1) the availability of a resource capable of eliminating the focus of a problem, and (2) the fact that a direct application of such resource might negatively affect elements that should be preserved. All other participants were sorted as unsuccessful schema generators $(N$ =16). This analysis showed that while $40.74 \%$ of successful schema generators later proposed a successful convergence solution to the tumor problem, only $12.5 \%$ of unsuccessful schema generators produced a convergence solution, $\chi^{2}(1, N$ $=70)=4.37, p=.0366, w=.25$. It thus seems that the actual production of an abstract schema, and not merely an attempt to generate it, is what allows for successful analogical transfer.

The second objective of Experiment 1 was to investigate whether the advantage of comparing two problems at test time depends critically on the provision of a second isomorphic target. The chosen strategy for addressing this issue consisted in assessing whether a transfer advantage could also be obtained by having participants compare the target problem with a nonisomorphic problem before attempting its solution. A chi-square test involving the comparing two disanalogous targets, the standard transfer, and the first principles control for disanalogous targets conditions failed to show an effect of the condition type on the proportion of convergence solutions to the Radiation problem, $\chi^{2}(2, N=210)=3.09, p=.2133$. Counter to our expectations, planned comparisons using Bonferroni adjustments showed that participants who compared two unrelated targets after receiving a base analog neither outperformed participants of the standard transfer condition in generating convergence solutions to the Radiation problem $(14.29 \%$ vs. $8.57 \%), \chi^{2}(1, N=140)=1.13, p=$ 
.2878 , nor outperformed participants in the corresponding first principles control $(14.29 \%$ vs. $5.71 \%), \chi^{2}(1, N=140)=2.86$, $p=.0908$. An analysis of the relation between schema abstraction and transfer helped assess the extent to which the failure of this last intervention to elicit successful transfer had to do with participants' failure to generate an abstract schema for the Radiation problem. The comparisons provided by participants who were asked to compare disanalogous targets were sorted into cases of successful schema generation $(N=15)$ and cases of unsuccessful schema generation $(N=55)$, according to the same criteria used for sorting the responses of participants who compared analogous targets. These results revealed that although $33.33 \%$ of successful schema generators later proposed a successful convergence solution to the radiation problem, only $9.09 \%$ of unsuccessful schema generators produced a convergence solution, $p=.031$ (Fisher's exact test). These data suggest that the poor transfer performance exhibited by participants who compared disanalogous problems is due to a failure of this task to elicit an appropriate abstract representation of the target problem to be solved. In Experiment 2, we focused on problem construction as another strategy potentially capable of helping learners capitalize on the late abstraction principle without requiring the provision of a second analogous target for every new problem they were to solve.

\section{Experiment 2}

The ability to construct structurally equivalent problems has been considered a stringent test of students' comprehension of different types of mathematics and algebra problems, as well as of their ability to relate them to the realm of real-world situations to which they can refer (see, e.g., Nathan, Kintsch, $\&$ Young, 1992). In a series of studies using probability problems, Bernardo (2001a, 2001b) demonstrated that the activity of constructing an isomorphic problem can be beneficial for later analogical transfer, on occasion even surpassing the problem-comparison task in efficacy. In an unpublished study described by Dunbar (2001), students who read fable-like stories with the task of generating analogous episodes were more likely to retrieve them during the processing of analogous stories lacking surface similarities. The above studies suggest that the activity of generating an analogous situation can be effective in directing participants' attention toward the structural features of the base situations being processed.

The fact that in studies such as Bernardo's (2001a) the construction of new base problems proved more effective for schema generation than the comparison of two externally provided analogous problems could reflect the potential of the invention activity to promote a relatively deeper and more active analysis of the problem structure. The construction of an analogous problem in a nonformal domain involves the postulation of entities that could play the roles of the base objects they replace. To continue with the Radiation problem, suppose that we begin by tentatively replacing the patient's stomach with the pit of a fruit, guided by a surface resemblance such as their spatial location inside an object. Next, we would have to relate the pit to another entity (e.g., a mold inside the pit), whose functionally relevant attributes (Keane, 1985) allow it to play in the fruit scenario the same role played by the tumor within the Radiation problem (i.e., threatening the rest of the fruit). The chosen element, in turn, constrains the selection of possible operators to those that would eliminate the focus of the problem when applied at the required intensity (e.g., by boiling the whole fruit), but at the cost of harming an element that needs to be preserved (e.g., the pulp). As illustrated by the above example, the derivation of an analogous target problem in a nonformal domain seems to demand a deep and systematic exploration of the relational structure of the problem that serves as a basis for problem construction.

Based on Kurtz and Loewenstein's (2007) and Gentner et al.'s (2009) idea that an encoding which highlights the underlying structure of the target analog should enhance access to analogous sources at test time, we reasoned that the activity of generating a relationally similar target analog could aid the retrieval of base analogs that were acquired under learning conditions that were not especially oriented toward prompting the extraction of structural features. The advantage of target construction over target comparison would lie in the fact that participants would be able to apply it to any potential target situation without the need to be externally provided with a second target analog.

\section{Method}

Participants and design A total of 210 undergraduate students at University of Comahue volunteered to take part in the study and signed an informed consent for participation. They were randomly assigned to each of the three conditions of the experiment (70 per condition). The dependent variable was the application of the convergence strategy to the target problem.

Procedure and materials Participants in the standard transfer condition (46 females and 24 males; $M_{\text {age }}=23.53, S D=3.06$ ) read the story The General (see Table 2 for a schematic presentation of the conditions and procedures of Experiment 2), framed between the same two distracter stories of Experiment 1 . After reading each of the three stories, they were asked the same question as in Experiment 1: "What critical insight allowed the problem in the story to be solved?" During the transfer phase, participants were asked to read the Radiation problem and to write down possible solutions. The written materials and the time allotted for each of the tasks were identical to those of the standard transfer condition of Experiment 1. 
Table 2 Problems and activities per condition in Experiment 2

\begin{tabular}{|c|c|c|c|}
\hline Condition & $\begin{array}{l}\text { Problems received during } \\
\text { learning phase }\end{array}$ & $\begin{array}{l}\text { Problems received } \\
\text { during transfer phase }\end{array}$ & Activity during transfer phase \\
\hline Standard transfer & The General & Radiation & Solving the Radiation problem \\
\hline Inventing an analogous target & The General & Radiation & $\begin{array}{l}\text { Inventing a second target analogous to the } \\
\text { Radiation problem and solving the } \\
\text { Radiation problem }\end{array}$ \\
\hline $\begin{array}{l}\text { First principles control for inventing } \\
\text { an analogous target }\end{array}$ & - & Radiation & $\begin{array}{l}\text { Inventing a second target analogous to the } \\
\text { Radiation problem and solving the } \\
\text { Radiation problem }\end{array}$ \\
\hline
\end{tabular}

The learning phase of the inventing an analogous target condition (42 females and 28 males; $M_{\text {age }}=22.50, S D=$ 2.75) was identical to that of the standard transfer condition. However, during the transfer phase, participants were asked to read the Radiation problem, and to create an analogous problem. The exact instruction was: "Two problems are analogous when they have the same structure but involve dissimilar objects. That is, while the objects change, they nevertheless maintain a comparable set of relations. Your task will consist in inventing a problem analogous to the tumor problem you have just read." As can be seen, this generic instruction did not provide any information about the underlying structure of the target problem. After being allotted 5 min for the task of creating a new problem, participants were allotted 10 more min to write down possible solutions to the Radiation problem.

As was done with the experimental condition of Experiment 1, a control condition (first principles control; 40 females and 30 males; $M_{\text {age }}=23.01, S D=3.11$ ) was implemented to assess the extent to which an eventual increase in the proportion of convergent solutions given to the tumor problem by participants who were asked to construct a new problem was in fact mediated by the retrieval of the source story. Participants in this last condition received the same materials and tasks given to participants during the transfer phase of the target construction condition, but without having received the critical base story and its solution during the learning phase of the experiment.

Scoring Following the same criteria as in Experiment 1, two raters blind to condition scored whether any of the responses given by each participant to the Radiation problem involved a convergence solution. Judges agreed in $98 \%$ of the cases, and solved cases of disagreement by discussion.

\section{Results and discussion}

The objective of Experiment 2 was to investigate whether the invention of a second target analog would increase the probability of retrieving distant analogs. The type of transfer condition yielded an effect on the proportion of convergence solutions to the Radiation problem, $\chi^{2}(2, N=210)=8.92, p=$
0116. In line with our expectations, planned comparisons using Bonferroni alpha correction revealed that participants in the invention condition outperformed participants in the standard transfer condition in generating convergence solutions to the Radiation problem $(25.71 \%$ vs. $10 \%), \chi^{2}(1, N=140)=$ $5.89, p=.0152, w=.21$. This increase in the proportion of convergent solutions cannot be attributed to an effect of target invention on the ability to solve the target problem from first principles, because participants who invented an analogous target without having previously received a base analog did not outperform participants in the standard transfer condition (10\% vs. $10 \%)$. Thus, the activity of constructing a novel unsolved target problem at test time seems to improve transfer by fostering the retrieval of the base story and its convergence solution.

To better assess the effect of analogical construction on analogical transfer, participants' success in generating a structurally equivalent problem was related to their performance in generating convergence solutions to the Radiation problem. Participants were sorted as successful problem constructors $(N=25)$ whenever the generated problem (1) involved the availability of a resource capable of eliminating the focus of a problem, and (2) stated the fact that a direct application of such resource might negatively affect elements that should be preserved. All other participants $(N=45)$ were sorted as unsuccessful problem constructors (see Table 3 for examples of the problems invented by participants). This classification revealed that although $48 \%$ of successful problem constructors later proposed a successful convergence solution to the tumor problem, only $13.33 \%$ of unsuccessful problem constructors produced a convergence solution, a difference that was highly reliable, $\chi^{2}(1, N=70)=10.11, p=.0015, w=.38$. It thus seems that it is the actual construction of an analogous problem and not merely an attempt to generate it that gives rise to the abstract schemas that are needed to facilitate analogical retrieval.

\section{General discussion}

Even though retrieving analogous problems from memory can lead to creative solutions for new problems, the fabric of our memory renders analogical retrieval infrequent in the absence 
Table 3 Examples of analogous target problems invented by participants of Experiment 2

1. The entire town was planning a protest against a sudden raise in the price of electricity. A prolonged and total interruption of consumption would make the company prone to rethinking the appropriateness of the raise. However, not using any electricity would involve threats to security, as well as the loss of refrigerated food.

2. A patient had a severe infection that needed to be controlled in less than an hour. There was a kind of antibiotic that could potentially control it, if an extreme dosage of it were immediately channeled into the blood flow. The problem was that injecting such an amount of antibiotic through any particular vessel would produce an irreversible damage to it.

3. There was an ink spot in a trouser. The spot could be removed by a solution of chlorine of the sufficient concentration. The problem was that a solution of the required concentration would also bleach the area surrounding the spot.

4. A car had fallen into a deep pit. Even though there were tows that had the sufficient strength to lift the car, attaching the tow to any single part of the chassis would break such part.

5. A scuba diver got locked inside a wreck and needed to be rescued immediately. A team of five professional divers would suffice to take him out of the wreck. However, such number of divers swimming together would capture the attention of sharks.

of surface similarities. Considerable effort has been spent in devising ways of promoting a structural codification of base analogs during their initial learning, so as to make them more accessible during later encounters with analogous but superficially dissimilar situations. However, given that they cannot be applied to already learned situations, Kurtz and Loewenstein (2007) and Gentner et al. (2009) have advocated shifting attention from the initial encoding of contents to the factors that affect the processing of target situations at the time of need. Specifically, they demonstrated that analogical retrieval can be boosted by asking participants to compare the target problem to a second isomorphic problem. Results from three conditions of the first experiment of this study replicated the advantage of comparing two isomorphic target analogs for transferring a base solution to a superficially dissimilar problem. Just as in Kurtz and Loewenstein (2007), however, at least a portion of this increase in the generation of convergence solutions can be attributed to a beneficial effect of target comparison in the participant's ability to solve the Radiation problem by first principles. Based on the particular features of the Radiation and The Fire Chief problems, we speculate that such portion of the target comparison advantage might have been due to a rerepresentation of participants' imprecise and hesitant understanding of radiation phenomena (e.g., of whether rays coming from opposite directions have an additive effect at their intersection) on the basis of their more precise and confident understanding of hydrodynamics. Given that a relatively more familiar domain in an analogical comparison naturally tends to be used as the basis for informing a situation whose understanding is relatively weaker (Gentner \& Gentner, 1983), participants' blind confidence about the additive nature of water supplies might have been used as a basis for resolving their ambiguous initial representation of overlapping rays in favor of an additive account.

Despite its success, the target comparison intervention falls short of representing a truly portable cognitive strategy because problem solvers require the provision of a second target analog for every potential problem they are to solve. To overcome this important limitation, in the remaining conditions of
Experiment 1 we assessed whether a transfer advantage could also be obtained by having participants compare the target situation to a "random" nonisomorphic problem bearing no ad hoc relation to the target problem. We had predicted that participants would naturally react to this assignment by focusing on similarities and differences at the level of the typical components of problems, such as goals, operators, and restrictions, which could lead to deemphasizing surface-level information and provide some abstract cues for interdomain retrieval. Counter to our expectations, comparing two nonisomorphic problems did not increase analogical transfer. It is conceivable, however, that having participants compare the target to multiple unrelated targets might yield a broader variety of retrieval cues, thus increasing the chances of focusing on the abstract features that are required to activate the critical base analog in the memory. In this line, studies on creative design (e.g., Christensen \& Schunn, 2009) showed that some of the gains obtained by providing participants with multiple retrieval cues are due to retrieving analogous cases.

In a further attempt to help learners capitalize on the late analogical abstraction principle without requiring the presentation of a second target analog, in Experiment 2 we tested the transfer advantages of asking participants to construct a novel problem analogous to the target, a task that had proven effective for eliciting the generation of abstract schemas out of a single base analog. We conjectured that the activity of constructing an analogous problem involving different elements would force participants to distill the abstract structure of the target, which could in turn enhance access to distant sources. In line with our prediction, participants being encouraged to construct a second target were more likely to retrieve a superficially dissimilar problem. Even though the successful construction of an analogous problem led to analogical transfer in a striking $50 \%$ of the cases, less than one third of the participants succeeded in generating an analogous problem. To date, most of the interventions involving problem construction were developed as ways of promoting and/ or testing the acquisition of concepts and procedures in mathematical subdomains such as arithmetic (Etheredge, Rudnitsky, \& Freeman, 1992; Lampert, 1986), algebra (Nathan et al., 1992), or 
probability (Bernardo, 2001a). Problem generation within these domains seems to be characterized by a great freedom for selecting the objects that instantiate the given quantities of the problem (e.g., apples, candies) as well as by a set of easily accessible actions that can potentially reinstantiate the mathematical operations represented by the base relations (e.g., replacing buying by another instance of adding, such as putting, and replacing selling by another instance of subtracting, such as removing). In contrast to generating analogous problems in mathematics, generating novel problems outside the realm of formal disciplines might require applying more aggressive mechanisms of conceptual slippage (Hofstadter \& FARG, 1995) as well as a more open and creative exploration of our (sometimes poor) knowledge about certain domains. To illustrate this, the generation of a problem like The Fire Chief on the basis of the Radiation problem could have begun by shifting the goal of the problem from destroying a tumor to suffocating a fire. Once this new goal was set, the remaining components of the problem had to be adjusted in a coordinated fashion, a process of multiple constraint satisfaction that can on occasions be rather taxing (Hofstadter, 1985; Ward, Smith, \& Finke, 1999). Although the replacement of rays by water jets is straightforward, it is less easy to think about what target element could fill the role of the surrounding tissues. It is thus not surprising that participants failed to carry out these replacements in a complete and coordinated manner. Despite these intrinsic difficulties, however, it is conceivable that training participants in the generation of analogous problems could render this strategy easier to implement. In view of the much greater portability of target construction as compared to the ad hoc provision of a second analogous target, it seems that any investment in enhancing this ability promises to pay off well.

A possible limitation of our findings concerns whether the results obtained with The General/Radiation problems would generalize to other problems as well. In favor of the generalizability of our findings, it is worth taking into account that a significant number of results originally obtained with these traditional problems was later replicated with other materials. As an example, the effect of surface similarities on retrieval, documented by Keane (1987) with these problems, was also observed with probability and combinatorics problems (e.g., Ross, 1987), and even with fable-like stories (Gentner et al. 1993), movie episodes (Trench \& Minervino, 2015, Experiment 1), and autobiographical memories (Trench \& Minervino, 2015, Experiment 2). Similarly, the success of interventions promoting forward transfer by means of comparing The General to The Fire Chief (Catrambone \& Holyoak, 1989) were later replicated using negotiation strategies (Gentner, Loewenstein, \& Thompson, 2003). This body of evidence on the generalizability of results obtained with these traditional problems suggests that our findings on the benefits of problem construction for backward transfer will likely generalize to other problems as well as to other types of analogs.
The most salient difference between the interventions aimed at improving the initial encoding of base analogs and those seeking to improve the encoding of target analogs at retrieval time lies in the fact that the latter, but not the former, may apply to a wealth of everyday situations learned under conditions that were not engineered to highlight their structural features. Despite the fact that our target-centered interventions did not explicitly promote an abstract encoding of base analogs, the results are silent about the extent to which the sources retrieved by participants of the late abstraction conditions had received an abstract processing during their initial encoding. However, most of the "enthusiasm" about interventions at recall time stems from the supposition that they allow retrieving suboptimally encoded knowledge in long-term memory (see Gentner et al., 2009; Loewenstein, 2010). For this possibility to make sense, further precision is required regarding (1) the different ways in which base encodings could be suboptimal, (2) the mechanisms by which such suboptimally stored knowledge could eventually be retrieved, and (3) the possible role of late abstraction in facilitating these particular kinds of analogical retrieval.

One slightly suboptimal way of encoding the base analogs could simply consist in having derived their abstract schema, but without having stressed its centrality relative to other more superficial aspects. Target interventions such as problem comparison or construction could potentially aid the retrieval of this kind of base analog. As Gentner et al. (2009) proposed, the representation of the target could include its generic structure plus the specific superficial features, or its generic structure but little about the specific superficial aspects. Although in the former case numerous surface matches will be retrieved, in the latter case there will be fewer surface matches to eventually out-compete and crowd out relational matches (Catrambone \& Holyoak, 1989; Gentner et al., 1993). Thus, diminished surface competition alone should be capable of accounting for an effect of analogical abstraction on backward relational retrieval. Additionally, the match between a schema and an example will be greater than the match between two analogous examples (Gentner et al., 2009). Simulation studies, using Forbus et al.'s (1995) MAC/FAC model of similarity-based retrieval, bear out the computational plausibility of this account.

Gentner et al.'s (2009) interpretation represents a plausible and parsimonious account of how the process of late abstraction could enhance access to base analogs whose structural traits were generated, but not especially highlighted. However, the success of various interventions aimed at inducing a more abstract encoding of source analogs suggests that participants are unlikely to encode the abstract structure of the source in ways that will neatly match the structure of the particular target they will have to solve. As an example, some participants might encode the goal of capturing the fortress as a case "overpowering an object by a force," a representation 
that could later be useful to describe the goal of destroying a tumor with rays in the Radiation problem (Gick \& Holyoak, 1983). But things would be different if instead of the Radiation problem, participants were tasked with figuring out how to bring a desired flow of water to a town while attending to the restriction that sending such flow of water through either of the available canals alone would flood the surrounding lands. For solving this particular target problem, it would have been better to conceptualize the goal of capturing the fortress as a case of, say, "carrying a resource to a destination." There are innumerable ways to encode exemplars (Hofstadter \& FARG, 1995; Markman \& Ross, 2003), and people can hardly guess which one will best fit a target problem that they do not yet know. In cases like this, late analogical abstraction could conceivably show a retrieval advantage over the raw processing of the target. Traditional models of analogical reasoning have postulated several rerepresentation mechanisms capable of discovering hidden identities between initially nonidentical elements (e.g., minimal ascension, Falkenhainer, Forbus, \& Gentner, 1989; decomposition, Gentner \& Wolff, 2000; or coactivation over distributed representations, Hummel \& Holyoak, 1997). Using a variant of minimal ascension, retrieval algorithms like ARCS (Thagard, Holyoak, Nelson, \& Gochfeld, 1990) gain access to base analogs having similar - but not identicalconcepts by means of probing long-term memory with memory cues derived from target elements by means of conceptual links such as superordination or part-whole relationships (e.g., the target concept destroy could activate a source containing the relation capture based on both being instances of overpowering). Given that connections between superordinates and their instances require fewer links than those between two particular instances (Gentner, 1989), interventions promoting late abstraction could in principle be capable of fostering the retrieval of base analogs whose structural encoding did not neatly match the structure of the target.

A third way in which the initial encoding of a base analog could be suboptimal would consist in storing the basic events of a base situation under a poor or wrong principle, as compared to the more proper principle under which the current target situation is being framed (Gentner et al., 2009). For instance, imagine that a psychology instructor wants to illustrate the well-known regression fallacy (Tversky \& Kahneman, 1974) with an example in which the son of an extraordinary soccer player displayed skills that, although competitive, were visibly inferior to those of his father. According to the topic of the lesson, people's natural tendency to construct causal explanations for these situations is ungranted, because such drops in performance could be more parsimoniously accounted for on purely statistical grounds. Now suppose that in an attempt to capitalize on her learners' prior knowledge, the instructor asked them to recall further examples. Faced with this task, a successful retrieval cue could consist of a structure where a descendent of a highperformance player did not display such level of performance, and where this drop has received a causal explanation. By means of promoting a shift between a particular type of extreme values (e.g., striking soccer skills) and more general ones (e.g., extreme values of any kind of professional achievement), interventions aimed at eliciting target elaborations could in principle be applicable to the retrieval of distant analogs that were indexed under a poor or wrong abstract principle.

In sum, while Gentner et al. (2009) have modeled the beneficial effect of late abstraction for retrieving a particular kind of suboptimally encoded sources (i.e., those cases where surface content has not been sufficiently deemphasized), there are other types of suboptimal encoding that may complicate subsequent retrieval (Loewenstein, 2010), and which deserve further inquiry. Dietrich (2000) advances several first-principles arguments favoring the thesis that analogical retrieval requires rerepresenting the to-be-retrieved source analogs, but he refers to the psychologists' skepticism regarding how to subject such hypothesis to empirical test (p. 265). Such empirical studies will require devising ways of assessing the depth of the base encodings without altering them in any way, something that might be difficult to achieve. Despite these difficulties, we believe that research efforts should be devoted to assessing whether suboptimally encoded sources can in fact get retrieved and the extent to which interventions such as comparison or invention can foster their retrieval.

Our results build upon a long tradition of empirical studies on the role of analogical retrieval in solving novel problems. Besides the importance of analogical problem solving within educational environments, interdomain analogizing serves as a basis for many other activities such as argumentation, explanation, creative design, or instruction. Future studies should address whether portable interventions to promote late abstraction can aid the retrieval of distant analogs in the service of these relevant activities.

Author Note This work was supported by the National Agency for Scientific and Technical Research (ANPCyT) under Grants PICT 2352 and PICT 2650, the National Council for Scientific and Technical Research (CONICET) under Grant PIP 0567, the National University of Comahue under Grant C108, and the Interamerican Open University under Grant PIB2013. A preliminary version of this study was presented at ANALOGY2013, Dijon, France, 2013.

\section{References}

Ahn, W. K., Brewer, W. F., \& Mooney, R. J. (1992). Schema acquisition from a single example. J Exp Psychol Learn Mem Cogn, 18, 391-412.

Bernardo, A. B. I. (2001a). Analogical problem construction and transfer in mathematical problem solving. Educational Psychology, 21, 137-150.

Bernardo, A. B. I. (2001b). Principle explanation and strategic schema abstraction in problem solving. Mem Cognit, 29, 627-633. 
Blanchette, I., \& Dunbar, K. (2001). Analogy use in naturalistic settings: The influence of audience, emotion, and goals. Mem Cognit, 29, $730-735$.

Catrambone, R. (2002). The effects of surface and structural feature matches on the access of story analogs. J Exp Psychol Learn Mem Cogn, 28, 318-334.

Catrambone, R., \& Holyoak, K. J. (1989). Overcoming contextual limitations on problem-solving transfer. J Exp Psychol Learn Mem Cogn, 15, 1147-1156.

Chen, Z., Mo, L., \& Honomichl, R. (2004). Having the memory of an elephant: Long-term retrieval and the use of analogues in problem solving. J Exp Psychol Gen, 133, 415-433.

Christensen, B. T., \& Schunn, C. D. (2009). "Putting blinkers on a blind man": Providing cognitive support for creative processes with environmental cues. In A. B. Markman \& K. L. Wood (Eds.), Tools for innovation: The science behind the practical methods that drive new ideas (pp. 48-74). New York, NY: Oxford University Press.

Clement, C., Mawby, R., \& Giles, D. (1994). The effects of manifest relational similarity on analog retrieval. Journal of Memory and Language, 33, 396-420.

Dietrich, E. (2000). Analogy and conceptual change, or "You can't step into the same mind twice". In E. Dietrich \& A. B. Markman (Eds.), Cognitive dynamics (pp. 265-294). Mahwah, NJ: Erlbaum.

Dunbar, K. (1995). How scientists really reason: Scientific reasoning in real-world laboratories. In R. J. Sternberg \& J. E. Davidson (Eds.), The nature of insight (pp. 365-395). Cambridge, MA: MIT Press.

Dunbar, K. (2001). The analogical paradox: Why analogy is so easy in naturalistic settings, yet so difficult in the psychology laboratory? In D. Gentner, K. J. Holyoak, \& B. Kokinov (Eds.), The analogical mind: Perspectives from cognitive science (pp. 313-334). Cambridge, MA: MIT Press.

Duncker, K. (1945). On problem-solving. Psychol Monogr, 58, 1-113.

Etheredge, M., Rudnitsky, A. N., \& Freeman, S. J. M. (1992). Writing math story problems: Children as authors. Paper presented at the Annual Meeting of the New England Educational Research Organization (May), Portsmouth, New Hampshire.

Falkenhainer, B., Forbus, K. D., \& Gentner, D. (1989). The structuremapping engine: Algorithm and examples. Artificial Intelligence, $41,1-63$.

Forbus, K., Gentner, D., \& Law, K. (1995). MAC/FAC: A model of similarity-based retrieval. Cognit Sci, 19, 141-204.

Gentner, D. (1983). Structure-mapping: A theoretical framework for analogy. Cognit Sci, 7, 155-170.

Gentner, D. (1989). The mechanisms of analogical transfer. In S. Vosniadou \& A. Ortony (Eds.), Similarity and analogical reasoning (pp. 199-242). Cambridge, UK: Cambridge University Press.

Gentner, D. (2003). Why we're so smart. In D. Gentner \& S. GoldinMeadow (Eds.), Language in mind: Advances in the study of language and thought (pp. 195-235). Cambridge, MA: MIT Press.

Gentner, D., \& Gentner, D. R. (1983). Flowing waters or teeming crowds: Mental models of electricity. In D. Gentner \& A. L. Stevens (Eds.), Mental models (pp. 99-129). Hillsdale, NJ: Erlbaum.

Gentner, D., Loewenstein, J., Thompson, L., \& Forbus, K. (2009). Reviving inert knowledge: Analogical abstraction supports relational retrieval of past events. Cognit Sci, 3, 1343-1382.

Gentner, D., Loewenstein, J., \& Thompson, L. (2003). Learning and transfer: A general role for analogical encoding. $J$ Educ Psychol, 95, 393-408.

Gentner, D., Rattermann, M. J., \& Forbus, K. D. (1993). The roles of similarity in transfer: Separating retrievability from inferential soundness. Cogn Psychol, 25, 431-467.

Gentner, D., \& Smith, L. (2012). Analogical reasoning. In V. S. Ramachandran (Ed.), Encyclopedia of human behavior (pp. 130 136). Oxford, UK: Elsevier.

Gentner, D., \& Wolff, P. (2000). Metaphor and knowledge change. In E. Dietrich \& A. Markman (Eds.), Cognitive dynamics: Conceptual change in humans and machines (pp. 295-342). Mahwah, NJ: Erlbaum.

Gick, M. L., \& Holyoak, K. J. (1980). Analogical problem solving. Cogn Psychol, 12, 306-355.

Gick, M. L., \& Holyoak, K. J. (1983). Schema induction and analogical transfer. Cogn Psychol, 15, 1-38.

Goldstone, R. L., \& Son, J. Y. (2005). The transfer of scientific principles using concrete and idealized simulations. The Journal of the Learning Sciences, 14, 69-110.

Goldstone, R., \& Wilensky, U. (2008). Promoting transfer by grounding complex systems principles. Journal of the Learning Sciences, 17 , 465-516.

Hofstadter, D. R. (1985). Metamagical themas: Questing for the essence of mind and pattern. London, UK: Viking.

Hofstadter, D. R., \& FARG. (1995). Fluid concepts and creative analogies: Computer models of the fundamental mechanisms of thought. New York, NY: Basic Books.

Hofstadter, D. R., \& Sander, E. (2013). Surfaces and essences: Analogy as the fuel and fire of thinking. New York, NY: Basic Books.

Holyoak, K. J. (1984). Analogical thinking and human intelligence. In R. J. Sternberg (Ed.), Advances in the psychology of human intelligence (Vol. 2, pp. 199-230). Hillsdale, NJ: Erlbaum.

Holyoak, K. J. (2005). Analogy. In K. J. Holyoak \& R. G. Morrison (Eds.), The Cambridge handbook of thinking and reasoning (pp. 117-142). Cambridge, UK: Cambridge University Press.

Holyoak, K. J., \& Koh, K. (1987). Surface and structural similarity in analogical transfer. Mem Cognit, 15, 332-340.

Hummel, J. E., \& Holyoak, K. J. (1997). Distributed representations of structure: A theory of analogical access and mapping. Psychol Rev, 104, 427-466.

Keane, M. T. (1985). On drawing analogies when solving problems: A theory and test of solution generation in an analogical problemsolving task. Br J Psychol, 76, 449-458.

Keane, M. T. (1987). On retrieving analogues when solving problems. $Q$ J Exp Psychol, 39, 29-41.

Kurtz, K. J., Boukrina, O., \& Gentner, D. (2013). Comparison promotes learning and transfer of relational categories. J Exp Psychol Learn Mem Cogn, 39, 1303-1310.

Kurtz, K., \& Loewenstein, J. (2007). Converging on a new role for analogy in problem solving and retrieval: When two problems are better than one. Mem Cognit, 35, 334-341.

Lampert, M. (1986). Knowing, doing, and teaching multiplication. Cognition and Instruction, 3, 305-342.

Loewenstein, J. (2010). How one's hook is baited matters for catching an analogy. In B. H. Ross (Ed.), The psychology of learning and motivation: Advances in research and theory (Vol. 53, pp. 149-182). San Diego, CA: Elsevier.

Markman, A., \& Moreau, C. (2001). Analogy and analogical comparison in choice. In D. Gentner, K. J. Holyoak, \& B. Kokinov (Eds.), The analogical mind: Perspectives from cognitive science (pp. 363 399). Cambridge, MA: MIT Press.

Markman, A., \& Ross, B. H. (2003). Category use and category learning. Psychol Bull, 129, 592-613.

Nathan, M. J., Kintsch, W., \& Young, E. (1992). A theory of algebraword-problem comprehension and its implications for the design of learning environments. Cognition and Instruction, 9, 329-389.

Nersessian, N. J. (1984). Faraday to Einstein: Constructing meaning in scientific theories. Dordrecht, Germany: Kluwer Academic.

Ross, B. H. (1984). Remindings and their effect in learning a cognitive skill. Cogn Psychol, 16, 371-416.

Ross, B. H. (1987). This is like that: The use of earlier problems and the separation of similarity effects. J Exp Psychol Learn Mem Cogn, 13, 629-639.

Ross, B. H., \& Kennedy, P. C. (1990). Generalizing from the use of earlier examples in problem solving. J Exp Psychol Learn Mem Cogn, 16, $42-55$. 
Ross, B. H., \& Kilbane, M. C. (1997). Effects of principle explanation and superficial similarity on analogical mapping in problem solving. J Exp Psychol Learn Mem Cogn, 23, 427-440.

Schwartz, D. L. (1995). The emergence of abstract representations in dyad problem solving. The Journal of the Learning Sciences, 4, 321-354.

Thagard, P., Holyoak, K., Nelson, G., \& Gochfeld, D. (1990). Analog retrieval by constraint satisfaction. Artificial Intelligence, 46, 259-310.
Trench, M., \& Minervino, R. (2015). The role of surface similarity in analogical retrieval: Bridging the gap between the naturalistic and the experimental traditions. Cognit Sci, 39, 1292-1319.

Tversky, A., \& Kahneman, D. (1974). Judgment under uncertainty: Heuristics and biases. Science, 185, 1124-1131.

Ward, T. B., Smith, S. M., \& Finke, R. A. (1999). Creative cognition. In R. J. Sternberg (Ed.), Handbook of creativity (pp. 189-212). Cambridge, UK: Cambridge University Press. 